\title{
Experiments or Theory? Aqueous fluids in the Deep Earth
}

\author{
JOËL BRUGGER ${ }^{1}$, BARBARA ETSCHMANN ${ }^{1}$, \\ Christopher GONZALEZ ${ }^{1}$, QIUSHI GUAN ${ }^{1}$, YUAN MEI $^{2}$, \\ PAOLO RAITERI ${ }^{3}$
}

${ }^{1}$ School of Earth, Atmosphere and Environment, Monash University, Clayton 3800, Victoria, Australia. joel.brugger@monash.edu

${ }^{2}$ CSIRO Mineral Resources, Kensington, WA 6151, Australia ${ }^{3}$ School of Life and Molecular Sciences, Curtin University, Perth, WA 6845, Australia

Supercritical aqueous fluids link subducting plates and the return of water, carbon, sulfur, and metals to the Earth's surface. Over the past decade, innovative theoretical thermodynamic extrapolations have extended our capacity to understand the role of aqueous fluids in the deep Earth. The new predictions suggest a more profound role for deep aqueous fluids than originally thought: for example, dissolution of carbonate in aqueous solutions at high pressure help balance the carbon budget in subduction zones, and polysulfide species, stable at high pressure, may form $\mathrm{Au}$ deposits rather than the reduced sulfur species stable at lower pressure.

With regard to metal complexes, these extrapolations are ultimately based on a large body of experimental studies; the vast majority were conducted at near ambient temperature and pressure; a reasonable amount investigated solutions to $\sim 300{ }^{\circ} \mathrm{C}, \mathrm{P} \leq 300$ bar; but few experimental data are available at pressures above $1 \mathrm{kbar}$, and hardly any reliable data is available beyond $10 \mathrm{kbar}$ for any metal complex.

The partial molar volume of dissolved species defines their relative stability at high pressure; yet partial molar volumes of most aqueous species are poorly constrained, because of the limited understanding of the pressuredependence of the energetics of these complexes. We will discuss how molecular dynamics can be used to address this fundamental limitation. Molecular dynamics (MD) simulations (calibrated using high quality experimental data) provide an independent control in determining the changes in molar volumes as a function of pressure and temperature. The data produced by MD will provide realistic properties in PT space that can be coupled to geodynamical simulations to investigate element transfer within complex tectonic environments, such as fluids expelled from subducting slabs to the surface, to help better constrain the deep Earth's volatile, redox and metal budgets. 